A Platinum Open Access Journal for Organic Chemistry

Paper

Free to Authors and Readers

DOAJ Seal

Arkivoc 2021, part vi, 256-267

\title{
Synthesis, analysis and study of sulfide-linked high functionality resin beads
}

Tibor Z. Kovacs, James A. Ramsden, lain P. Thomas, and John M. Brown*\#

The Dyson Perrins Laboratory, University of Oxford, South Parks Rd., Oxford OX1 3QY, UK

\# Present address: CRL, Oxford Chemistry, University of Oxford, Oxford OX1 3TA, UK

Email: John.brown@chem.ox.ac.uk

In appreciation of the career-long commitment of Philip Hodge to the advancement of organic polymer chemistry

Received 08-28-2021

Accepted $10-08-2021$

Published on line $10-24-2021$

\section{Abstract}

A synthetic method for linking a spacer chain to highly functionalized chloromethylated resin beads was developed and applied in distinct projects, studying the incorporation of small peptide chains, the ion-pairing ability of charged beads and the potential for an inter-bead Diels-Alder reaction. ${ }^{13} \mathrm{C} N M R$ and photomicroscopy were the main experimental techniques utilized for these applications.

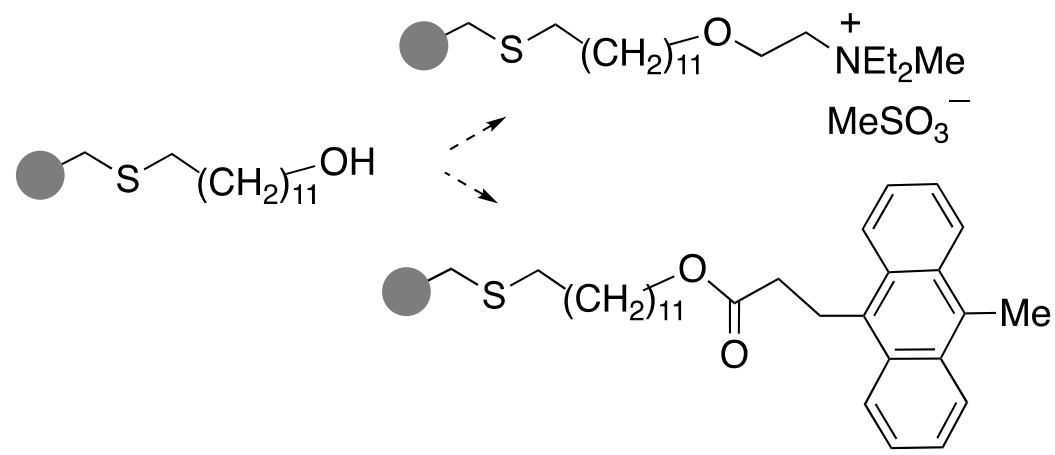

Keywords: Polymer beads, ${ }^{13} \mathrm{C}$ NMR, coupling, ion pairing, Diels-Alder, photomicroscopy. 


\section{Introduction}

This manuscript provides a fuller account of work carried out in the late 1990s and previously published as two short communications. It represents research conducted during the relatively brief period when there was a strong interest in using combinatorial chemistry for parallel synthesis in drug discovery, providing an incentive for collaborations with the academic community in that field. These are the main contributions of the corresponding author's group to a field where Phil Hodge has made such wide-ranging and significant contributions.

The initial involvement of the Oxford research group in polymer chemistry arose from earlier interest in micellar catalysis, an area of research that was extensively studied during the 1970 s. $^{1,2}$ The general aim was to synthesise surfactants as precursors for functional micelles that had the potential for distinct properties or shown divergent chemistry. Through a collaboration that required the substantial expertise in the field of Bunton's group in UCSB, the first example of stereoselective micellar catalysis was recorded, through synthesis of a cationic trimethylammonium surfactant carrying an L-histidine group in the side chain. ${ }^{3,4}$ The active catalyst crystallised and later both diastereomers were separated by chromatography. It was shown to be the $(S, S)$-diastereomer that preferentially reacted with the $(S)$-enantiomer of substrate. ${ }^{5}$ Changes in the internal structure of micelles through specific hydroxylation of the carbon chain were observed by ${ }^{13} \mathrm{C}$ NMR relaxation techniques. ${ }^{6} \mathrm{~A}$ single paper was directed to the study of micellar polymer chemistry in this period, through the synthesis of polystyrene beads with a high loading of cationic surfactant sidechains, based on chloromethylated Merrifield resin 1 (Figure 1). The product was hydrophilic, swelled by $40 \%$ in water, and catalysed the $O$-alkylation of sodium 2-naphthoxide 2 by benzyl bromide. ${ }^{7}$ In pure water the reaction gives $86 \%$ of the $C$-alkylation product 3 ; in the presence of a soluble cationic surfactant $O$-alkylation to 4 is predominant. ${ }^{8}$ The polymer beads behaved in a similar manner giving $98 \%$ of 4 and they also enhanced the reaction rate. These observations lead to the conclusion that the micellar polymer functioned effectively as a heterogeneous phase transfer catalyst in water. Related examples published in that period with different polymer micelles support that interpretation. ${ }^{9,10}$ At that time there was a general level of interest in resin bead supported catalysts; ${ }^{11}$ this encouraged our synthesis and application of a polymer-supported hydrogenation catalyst. $^{12}$

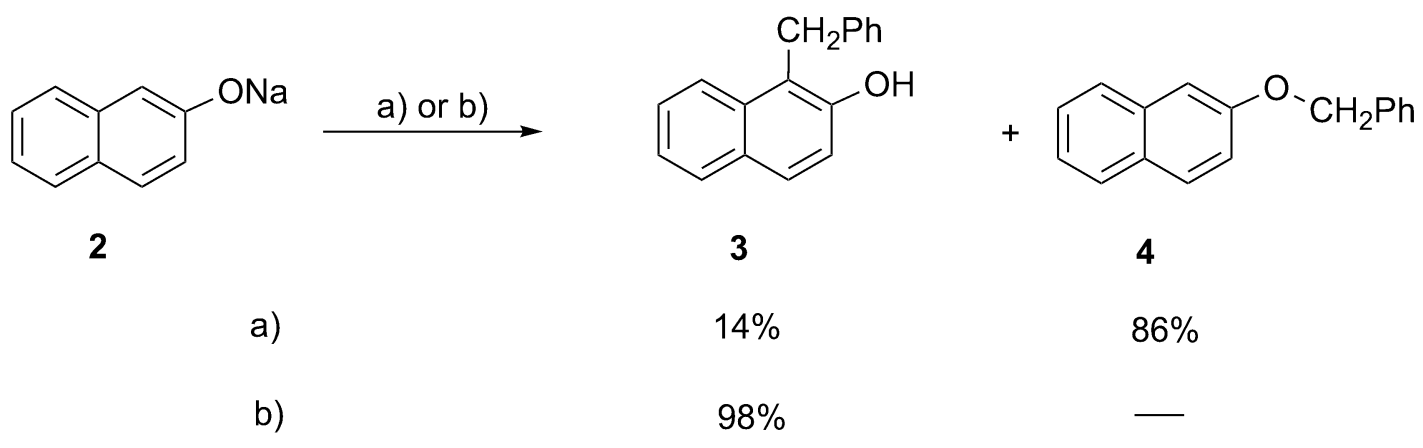

Conditions: a) $\mathrm{H}_{2} \mathrm{O}, 20^{\circ} \mathrm{C}$.

b) $\mathrm{H}_{2} \mathrm{O}, 20^{\circ} \mathrm{C}$; catalytic

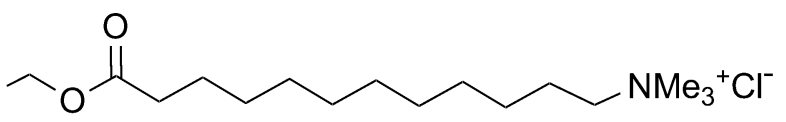

1: $\quad=2 \%$ crosslinked PS.

Figure 1. Heterogeneous phase transfer catalysis in water. 
The rapid development of catalytic asymmetric synthesis at that time diverted research group resources away from the field for over a decade during which time major contributors to the organic polymer field became established and the links between synthetic organic chemistry and polymer chemistry were strengthened. ${ }^{13,14}$ The emergence of combinatorial methods in the pharmaceutical industry in the 1990s and more specifically their interest in "bead chemistry" provided the encouragement (and the resources) for further effort. Two separate research areas topics involved the Oxford group during this period. For the first, resin beads with a high loading of a spacer group were synthesised and utilised in small peptide synthesis. ${ }^{15}$ The second area addressed the possibility of pairwise chemical bonding at the interface between polymer beads, and methods for defining its occurrence. ${ }^{16}$ Fuller discussion of both topics is provided in this paper.

\section{Results and Discussion}

\section{Synthesis and characterization of polymer beads with spacer groups.}

The commercial availability of $63 \%$ functionalized chloromethylated $2 \%$ crosslinked Merrifield resins provided the basic material for the project. Spacer groups that increased local mobility of the pendant chains and the quality of NMR analyses were incorporated, by standard $S_{N} 2$ reaction of solvent-swollen polymer with thiolate nucleophiles. The thiolate coupling step was developed by trial and error as the most effective way of introducing a flexible sidechain in high yield under our conditions. Starting with the known thioacetate $\mathbf{5}^{17}$ dihydropyran (DHP) protection gave thiol 6 that reacted directly with chloromethylated polymer to give the functional derivative $\mathbf{7}$, that was converted to $\mathbf{8}$ by mild acid treatment, which removed the dinitrophenyl group and left a free hydroxyl group at the chain terminus (Figure 2).

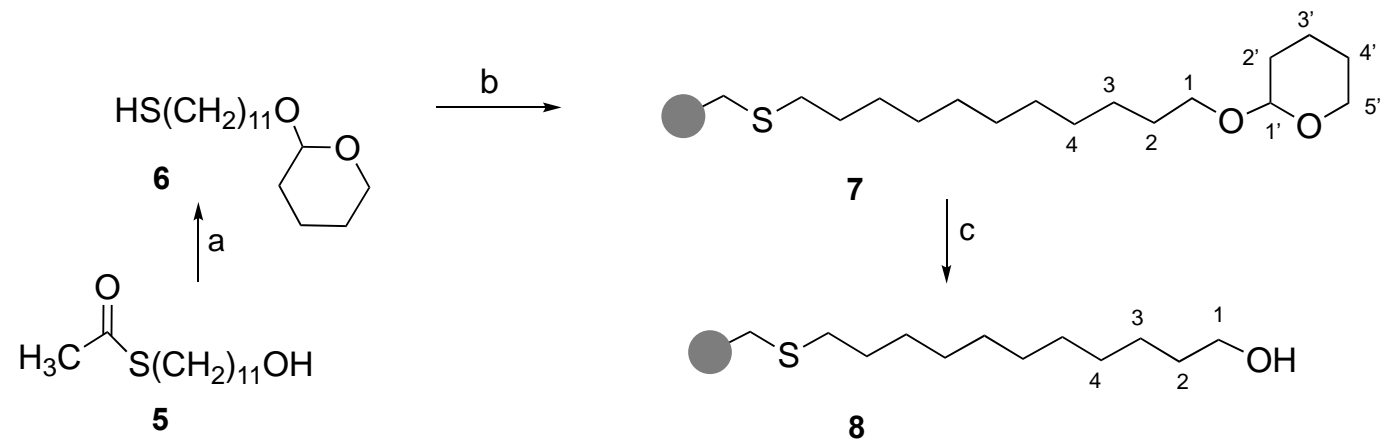

Reagents: a) DHP, $\mathrm{TsOH}, \mathrm{CH}_{2} \mathrm{Cl}_{2}$, then $\mathrm{NaOH}, \mathrm{H}_{2} \mathrm{O}, \mathrm{MeOH}$; b)

$\left.-\mathrm{CH}_{2} \mathrm{Cl}, \mathrm{NaH}, \mathrm{THF} ; \mathrm{c}\right) \mathrm{HCl}, \mathrm{MeOH}, \mathrm{CH}_{2} \mathrm{Cl}_{2}$.

Figure 2. The synthesis of function-bearing polymer bead $\mathbf{8}$ from reactant $\mathbf{5}$.

To be useful further, the terminal region of the chain needs to experience a fluid environment, and this was tested by the ${ }^{13} \mathrm{C} \mathrm{T}_{1}$ spin-lattice relaxation studies shown in Figure 3. Under the conditions used, the polymeric species $\mathbf{7}$ and $\mathbf{8}$ These observations led to the conclusion that the functional polymers $\mathbf{7}$ and $\mathbf{8}$ showed satisfactory mobility in the region of the solvent interface when suspended in $\mathrm{CDCl}_{3}$. Under the conditions used, the polymeric species showed $T_{1}$ relaxation times between 0.4 and 1 sec., whilst the precursors gave $T_{1}$ values of between 1.2 and $2 \mathrm{sec}$. Spin-lattice relaxation times are temperature-dependent and for a specific carbon atom they are also dependent on the number of directly attached hydrogens and inversely dependent on the spectrometer frequency. ${ }^{18,19}$ After making appropriate adjustments, the $T_{1}$ values 
for C1, C2 and C3 of 7 shown in Figure 3 are broadly comparable with those observed for the equivalent carbons of polyglycol monoethers close to the hydroxyl terminus in $\mathrm{CDCl}_{3}$ solution. ${ }^{20}$ For poly(n-alkyl methacrylates), $\mathrm{T}_{1}$ determinations in $\mathrm{CDCl}_{3}$ demonstrate that the mobility of the side-chain alkyl moiety attenuates markedly with increasing distance from the terminal methyl group, ${ }^{21}$ as is observed in the present case with increasing distance from the terminal $\mathrm{OH}$ group in precursor $\mathbf{1}$ and polymer 2 . Hence mobility information obtained for a polymer in $\mathrm{CDCl}_{3}$ solution correlates well with that obtained here for polymer beads swollen in the same solvent.

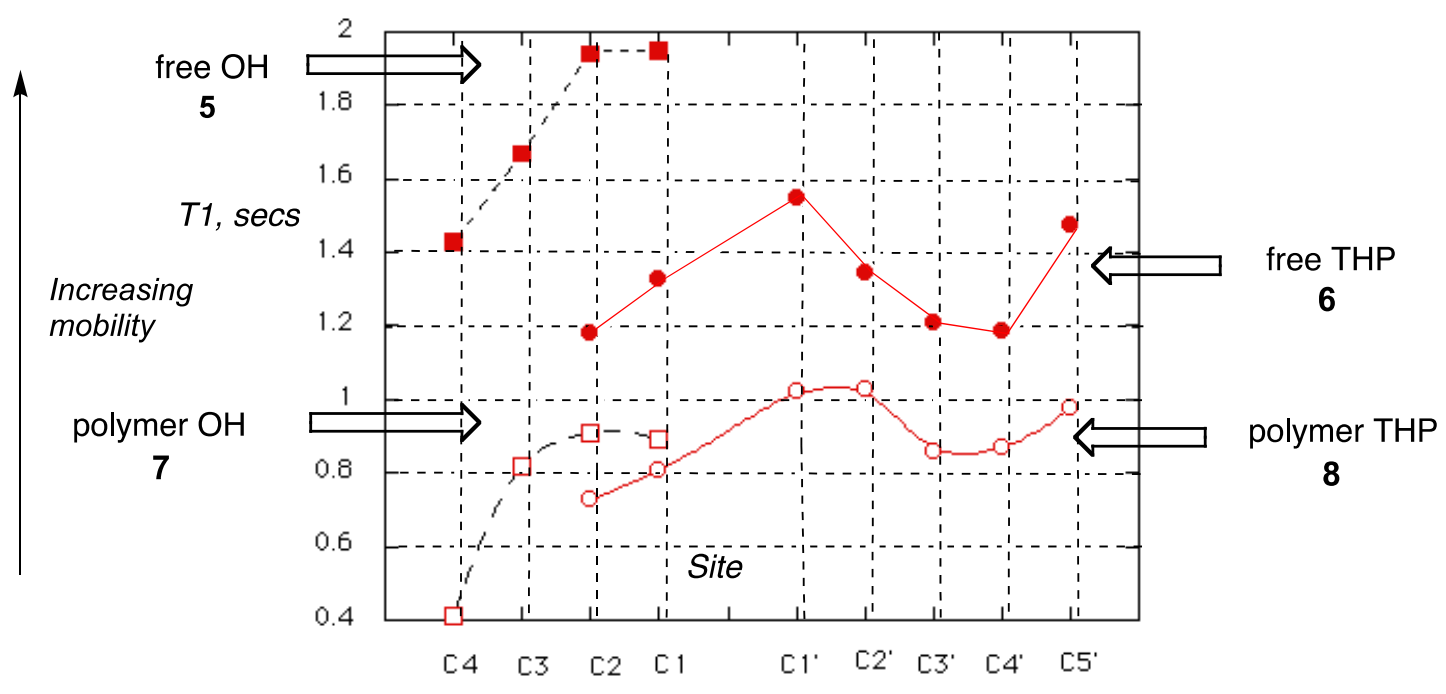

Figure 3. $T_{1}$ relaxation data for polymer beads 7 and 8 in comparison to the precursors 5 and 6 (THP = tetrahydropyranyl).

With this information in hand, methods for high yield coupling of amino acids to the spacer terminus were pursued. Optimum conditions were found by trial and error; one set was superior to all other methods attempted. For this, it was found that Fmoc-protected aminoacyl fluorides were coupled to the free hydroxyl group of the resin in high yield, using the coupling agent DCC/HOBt (di-cyclohexylcarbodiimide / hydroxybenzotriazole). ${ }^{22}$ For comparison, the corresponding aminoacyl chlorides gave unsatisfactory yields in the same coupling reaction. The second coupling step of the amino-acid side chain attachment was accompanied by the known side reaction of dioxopiperazine formation, ${ }^{23}$ that reduced the number of active sites by ca. $25 \%$, but without hindering the remainder. By following the coupling method protocol stepwise, a tetrapeptide was assembled on the functional polymer with an average $95 \%$ incorporation of amino acid on active sites over the four individual steps. The resulting polymer-bound tetrapeptide was analyzed by ${ }^{13} \mathrm{C}$ NMR; the optimum conditions required DMSO- $d_{6}$ as solvent and an elevated temperature of $323 \mathrm{~K}$. The $\alpha-$ carbon of the amino acid residues resonated in the 44-64 ppm region and showed separated ${ }^{13} \mathrm{C}$ signals for all four component amino acids. (Figure 4). The observed signal half-widths were between 20 and $40 \mathrm{~Hz}$, reflecting increased mobility of the residue with increasing distance from the polymer backbone. 


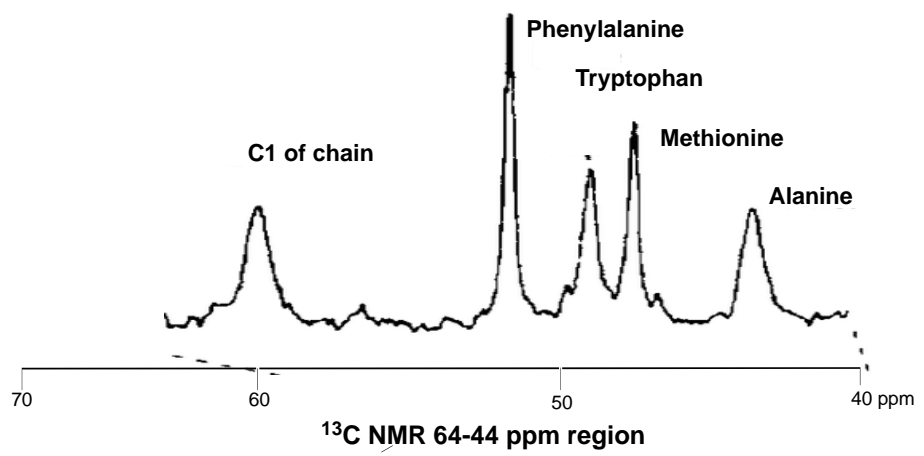

Figure 4. ${ }^{13} \mathrm{C}$ NMR spectrum of the $\alpha$-carbon region of the pendant amino acid chain -Ala-Trp-Met-Phe compared with $\mathrm{C} 1$ of the linking group.

The incorporation of a variety of functional groups attached to the C11 thiolate-bonded spacer 8 via activation or displacement of the hydroxyl group was then carried out, the choice of linking group being based on the need to maximize the molar equivalence of resin catalysts and reagents and optimize NMR experiments as far as possible. An observed limitation was that reactions with electrophilic methylating agents led to competing methylation of the sulfide group that resulted in cleavage of the spacer, reducing the yield of an otherwise clean reaction by up to $30 \%$. For this reason, later Oxford work used the then commercially available polyglycol-spaced resin beads. ${ }^{24}$

Within three years of completion of the work described here, the impetus from combinatorial chemistry in Pharma for more rapid and smaller scale analysis of polymer-supported reactions quickly led to enhanced NMR methods of functional bead analysis. The complete NMR analysis of a single polystyrene bead in a nanoprobe using ${ }^{1} \mathrm{H}$ NMR with MAS (magic-angle spinning) could be achieved. ${ }^{25,26}$

\section{A. Application to Inter-bead association by surface charge attraction to form macroscopic ion-pairs.}

Given the ability to synthesize functional sidechains on polymer beads it was of interest to observe their macroscopic chemistry. Ion-pairing between resin beads with opposite charges associated with the terminal functional group offered a simple starting point. Both the cationic and anionic polymers were synthesized from the same precursor 8 according to Figure 4. The cationic component 9 was characterized with $\mathrm{CH}_{3} \mathrm{SO}_{3}{ }^{-}$as its counterion, giving an independent $\mathrm{CH}_{3}$-signal in the ${ }^{13} \mathrm{C}$ NMR spectrum that allowed checking the signal strength against the $\mathrm{N}$-methyl head group for complete ion exchange. The anionic beads $\mathbf{1 0}$ were synthesized by a phosphorylation route, following a literature procedure, and isolated as the lithium salt. ${ }^{27}$ Direct visual analysis required dye staining of beads and in the simplest case involvement of a charged dye in one component of the ion-pair. The cationic dye methylene blue $\mathbf{1 1}$ is soluble in common organic solvents and was used in this study, with the components synthesized according to Figure 5. It was confirmed that treatment of the mesylate salt $\mathbf{9}$ with $\mathbf{1 1}$ to exchange the cationic counter-ion caused a deep blue staining of the beads. On mixing and stirring the colorless anionic beads $\mathbf{1 0}$ rapidly aggregated with the stained cationic beads derived from 9 by treatment with 11. After washing with water, mixed clusters and alternating blue and colorless chains of 5-8 beads were observed under a microscope, behavior that was not shown in control experiments where the opportunity for bead-bead ion-pairing was absent. This positive result encouraged the more challenging search for chemical bonding, between beads carrying linking groups that are designed to encourage a coupling reaction. 


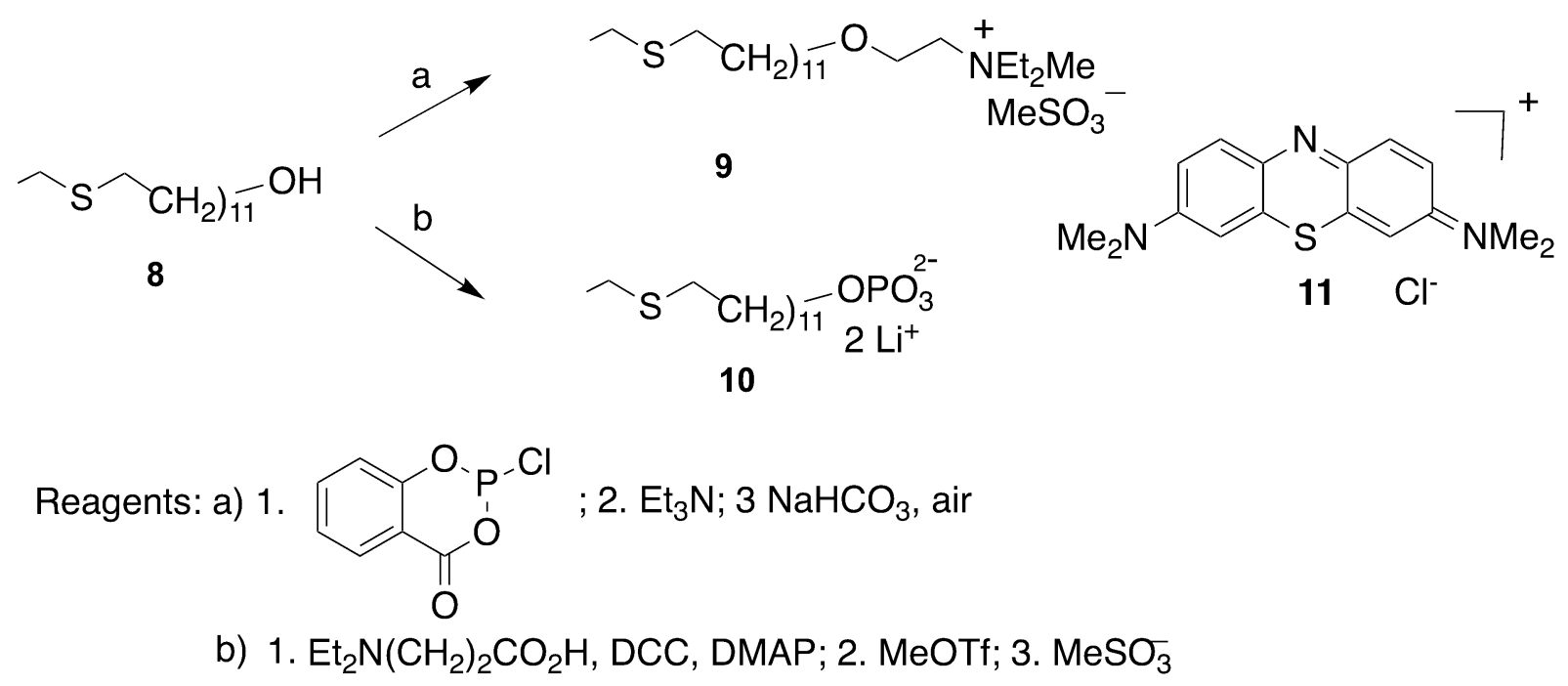

Figure 5. The synthesis of polymer beads for the study of inter-bead ion pairing.

\section{B. Application to a Diels-Alder reaction between diene and dienophile functional beads.}

The choice of a Diels-Alder reaction for exploring inter-bead reactivity was based on several favorable factors. Reactions can be rapid with quantitative formation of a single product and a relatively small response of reaction rate to solvent polarity. Furthermore, the internal symmetry of an anthracene-based reactant avoided the formation of stereoisomers in the cycloaddition step. ${ }^{28,29}$ Dienophile and diene functional groups were both successfully coupled to polymer beads giving $\mathbf{1 2}$ and $\mathbf{1 3}$ respectively (Figure 6). By the same criterion, the anthracene-bearing polymer 13 reacted to completion with maleimide, based on the ${ }^{13} \mathrm{C} N M R$ spectrum of the product. The dienophile polymer 12 reacted to completion with cyclopentadiene. Finally, and crucial to the development of the chemistry described below, the diene beads could be stained with by DielsAlder addition of the deep red compound 14, synthesized by incorporating a maleimide side chain in the commercial dyestuff Disperse Red 1. Stable red beads of $\mathbf{1 5}$ were observed when this cycloaddition was carried out to the extent of ca. $1 \%$ of the available diene sites. Further experiments demonstrated that both diene and dienophile beads aggregated in cyclohexane but were fully dispersed in DMF. Since covalent bonding between beads was novel, rigorous procedures and control experiments were needed. On this basis protocols for observing heterotopic association (12-15) and distinguishing this from homotopic association (12-12 or 15-15) were needed together with techniques for micro-manipulation and microphotography (see the Experimental Section for details). In a preliminary test, approximately equal quantities of diene resin $\mathbf{1 5}$ and maleimide resin $\mathbf{1 2}$ were briefly sonicated together and then suspended in toluene. After 3 days there was a considerable level of aggregation that persisted on transfer to DMF. Such aggregation was not observed during the corresponding control experiment involving the mixing of 15 and 16, the saturated analog of 12, where the mixture remained fully dispersed on suspension in DMF with no evidence for inter-bead reaction. 


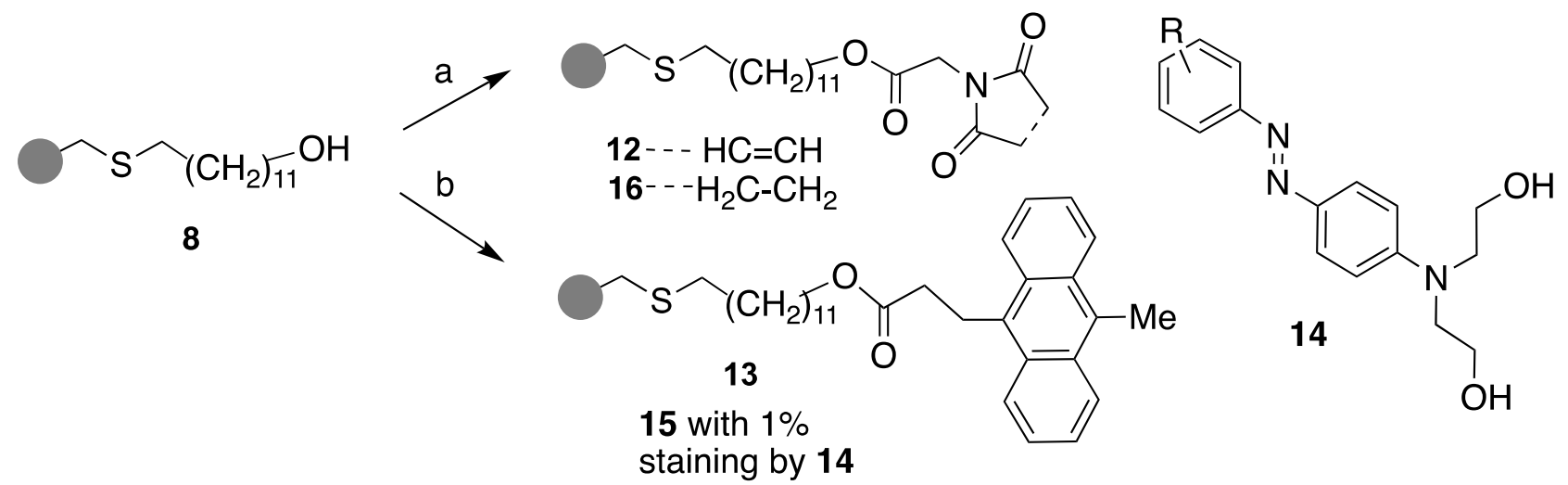

Figure 6. a,b: Coupling of polymer 8 with acid chlorides to give 12, 13 or 16; Reagents for a, b: $\mathrm{Py}, \mathrm{CH}_{2} \mathrm{Cl}_{2}$, Room temperature.

Further experiments demonstrated that both diene and dienophile beads aggregated in cyclohexane but were fully dispersed in DMF. Since covalent bonding between beads was novel, rigorous procedures and suitable control experiments were developed. Working on this basis, protocols for observing heterotopic association (12-15) and distinguishing this from homotopic association (12-12 or 15-15) were needed together with techniques for micro-manipulation and microphotography (see the Experimental Section for details). In a preliminary test, approximately equal quantities of diene resin 15 and maleimide resin 12 were briefly sonicated together and then suspended in toluene. After 3 days there was considerable aggregation that persisted on transfer to DMF. Similar aggregation was not observed during the corresponding control experiment involving 15 and 16, the saturated analog of 12, where the mixture remained fully dispersed on suspension in DMF.

The following additional observations were made in the course of studying the inter-bead reaction:

(a) The extent of aggregation and the average size of aggregates increased over time. Even without mechanical intrusion the proportion of heterotopic (diene plus dienophile 12-15) contacts was significantly higher than statistical, the proportion of homotopic (diene plus diene 15-15 or dienophile plus dienophile (1212) contacts was likewise significantly lower than statistical expectation.

(b) The nature of the interface was distinct for heterotopic contacts when compared to homotopic contacts. For the latter case, the point of contact was not well defined so that two or three beads made visible rolling movements on visual examination, the stable state of most such three-bead aggregates being triangular. In contrast, heterotopic association between beads occurred at a fixed point and the interaction was stable. Alternating linear three and four-bead aggregates were observed, and rarely higher states of aggregation. After separation of heterotopic bead aggregates on a microscope slide they were photographed and a sample of the images is shown in Figure 7.

(c) By carrying out the staining procedure after inter-bead reaction, it was verified that heterotopic interactions were uniquely responsible for the distinctive stable linear trimers and tetramers. Although these aggregates were just robust enough to be mechanically manipulated, their fragility is evident from the loss of adhesion after evaporation of all solvent DMF and consequent shrinkage of the beads.

(d) Heterotopic aggregates formed in DMF were not broken down in a $1 \mathrm{M}$ solution of maleimide in DMF after $20 \mathrm{~min}$. 
(e) The surface contact area is small, estimated to be in the region where $10^{4}$ molecules of the diene and dienophile components have the potential to undergo Diels-Alder reaction. ${ }^{16}$ This was considered to be below the sensitivity range of instrumentation available to the authors at that time.

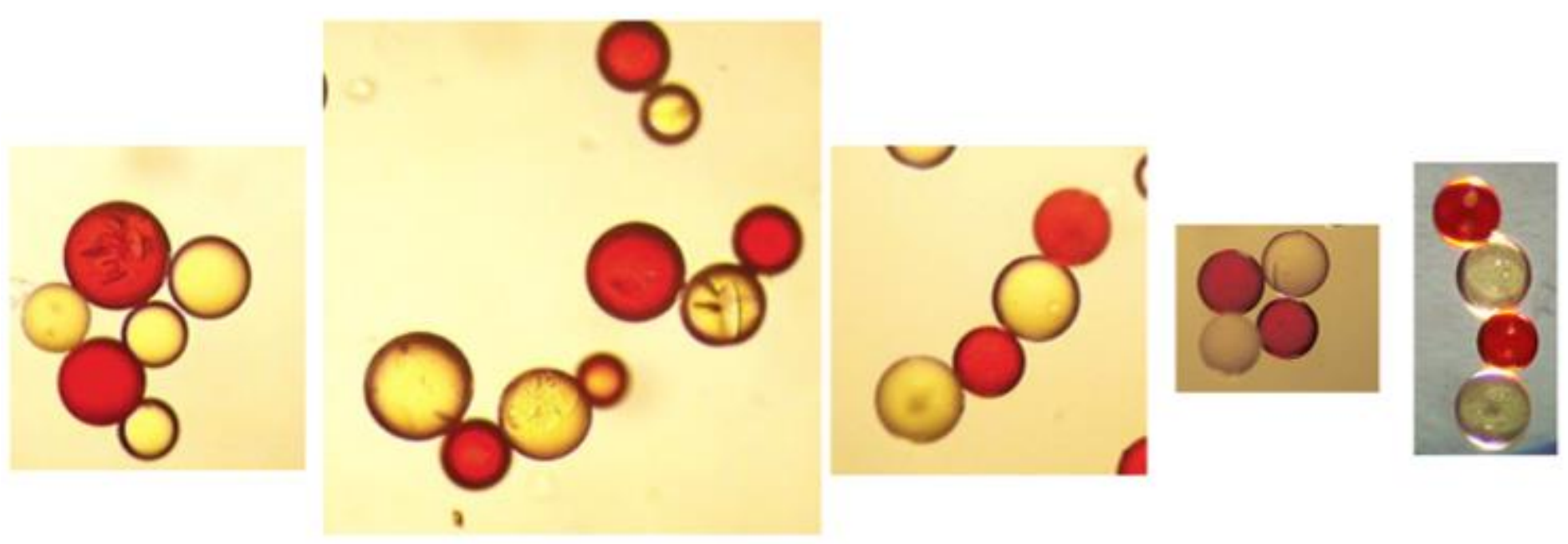

Figure 7. Formation of stable heterotopic clusters of diene and dienophile beads taken from different samplings. The sizes of the beads utilised here were estimated to be in the range of $50-100 \mu$ after the completion of side-chain synthesis.

\section{Conclusions}

A thiolate-bonded spacer had been developed for the synthesis of polymer beads bearing high levels of active functionality. The $\alpha \omega$-hydroxysulfide used here had been previously applied extensively for the adsorption of gold salts at monolayers, following the initial seminal paper by Bain and Whitesides. ${ }^{30}$ The application as a spacer group in this work served the intended purpose admirably, but a vulnerability to C-S cleavage of the linker chain by reaction with powerful anionic nucleophiles or powerful oxidants was recognized during the course of research. Long term storage did not lead to any diminution of activity. Within three years of the initial publication, highly functionalised polystyrene beads carrying polyglycol spacers became commercially available and these were subsequently preferred. The aim was to demonstrate controlled bonding between two macroscopic objects involving a limited number of reactions at their interface, as demonstrated here.

In 2001, Sharpless and co-workers provided a milestone for organic chemists, introducing "Click Chemistry". ${ }^{31}$ Their detailed review of available synthetic methodology was designed to facilitate drug discovery, and based on the premise that a relatively small number of reactions involving the coupling of two components would provide access to a majority of desired molecular frameworks. Cycloadditions featured prominently in their list and was exemplified in practice by Cu-complex catalysed cycloaddition of azides and alkynes. $^{32}$ The Diels-Alder reaction has been widely used subsequently in Click Chemistry as one of the essential structural building blocks, largely targeting small molecules of synthetic, biological or medical interest. ${ }^{33}$ These include the synthesis of 2-pyrazinone scaffolds and the conjugation of oligonucleotides ${ }^{34,}, 35$. A stated objective for Click Chemistry was to encourage Pharma to reduce their level of reliance on Combinatorial Chemistry, and this was heeded. The change in emphasis influenced academic priorities to the extent that interest in the development of spacer linked polymer beads declined after the eary 2000 s. 
Ironically, the polymer community adopted Click reactions widely and in a variety of ways. Click Chemistry was applied to the synthesis of dendrimers, ${ }^{36}$ electrooptic polymers, ${ }^{37}$ or RAFT (Reversible Addition Fragmentation Chain Transfer) polymers, ${ }^{38}$ by means of dipolar cycloaddition or a Diels-Alder reaction. In two cases the maleimide-anthracene condensation described here has been directly utilized in polymer chemistry for related purposes. ${ }^{39,40}$

\section{Experimental Section}

General. Initial work was guided by the commercial availability of a range of chloromethylated polystyrene beads (Fluka). The standard function-bearing Merrifield resin was selected (2\% crosslinked, $37-74 \mathrm{~mm}, 63 \%$ chloromethylation) on the grounds of its physical robustness over smaller beads and its high density of functionality. Reactions that involved coupling between the spacer groups of beads and reactants were expediently carried out under solvent, in a gently rotating Rotavapor-M held at ambient temperature and pressure.

Acquisition of ${ }^{13} \mathrm{C}$ NMR spectra: ${ }^{13} \mathrm{C}$ NMR spectra were routinely recorded on a Varian Gemini 200 (50.3 MHz), occasionally at higher field strength (Bruker DPX 400 (100.6 MHz) or Bruker AM 500 (125.8 MHz). Polymer samples were prepared by placing an appropriate quantity of polymer beads (ca. 120-130 mg) in a $5 \mathrm{~mm} \mathrm{NMR}$ tube, with small amounts of solvent until the polymer was fully swollen and 2-4 $\mathrm{mm}$ of excess solvent remained. Suspensions in $\mathrm{CDCl}_{3}$ or $\mathrm{DMF}-\mathrm{d}_{8}$ gave superior spectra. The tube was then vibrated either by using a sonicator, or by gently tapping the tube on a wooden surface until no air bubbles were visible and the suspension appeared homogeneous. The sample was then allowed to settle for a minimum of 10 min. before being placed in the spectrometer. It was found that at $50 \mathrm{MHz}$ acceptable spectra were obtained in ca. 30 min., and a high S/N after 1-3h. With the exception of very broad signals for aromatic carbons in the $125 \mathrm{ppm}$ region, relevant peaks arising from the spacer carbons were well resolved with a linewidth of 20-50 $\mathrm{Hz}$. $\mathrm{T}_{1}$ measurements were carried out with a relaxation time of $12 \mathrm{~s}$ and mixing times of $1 \mathrm{~ms}$ to $13 \mathrm{~s}$.

Bead Staining: To facilitate the analysis of bead-bead interaction by microscopy, the two bead types were differentiated by staining. Diene beads (13) were pre-stained by treatment with a $1 \%$ THF solution of the dienophile derivatized with Disperse Red I dye (14) for 1 hour at ambient temperature. The beads were washed with copious THF; the recovered beads (15) were clearly stained red. No decolorization of the beads is observed over many months. The ${ }^{13} \mathrm{C}$ NMR spectrum of the stained beads $\mathbf{1 5}$ lacks resonances that can be assigned to the dye fragment, i.e. the number of diene sites quenched with dye is very small.

Sample Preparation: Dry samples of $\mathbf{1 2}$ and $\mathbf{1 5}$ were mixed in Eppendorf tubes and swelled in DMF. Samples were sonicated for $\sim 15$ s to ensure good mixing of the two bead types. Samples were centrifuged at 6500 rpm for between 0 and 75 minutes. Centrifuging reduced relative motion of the beads possibly facilitating interbead Diels-Alder reactions. Samples suitable for microscopy were prepared by first placing 2 to 3 drops of water on a microscope slide and dropping a small sample of beads in DMF into the water from a pipette. The water droplet was spread carefully over $\sim 2-3 \mathrm{~cm}$ using the tip of a pipette. The beads disperse in water and have low mobility.

Bead Observation: The set-up of digital camera linked to bench-top microscope permitted physical observations of solvent swelling, bead fragmentation and surface adhesion to be readily followed and analyzed. If required, the manipulation of single beads was achieved using micromanipulators.

Bead Counting: For each point in a timed sequence, an aliquot was removed from the reaction vessel, and 
three separate samples containing ca. 500-1000 beads were transferred to microscope slides. A microscope slide was placed on a small sheet of graph paper with a highlighted $1 \mathrm{~cm}^{2}$ grid square subdivided into twentyfive $4 \mathrm{~mm}^{2}$ areas. The beads within the $1 \mathrm{~cm}$ grid were counted with the aid of hand-held tally counters. Counting was performed in 3 "passes" over the sample in 15-20 mins. There was no significant displacement of free and aggregated beads over this time. Three different samples were counted for each experiment. The accuracy of counting was checked on 2 or 3 occasions by recounting the same sample; repeat counts were well within $5 \%$ of each other.

\section{Data Acquisition:}

Pass 1: The number of red and white beads was counted.

Pass 2: The numbers of red-red, white-white and red-white interactions were counted. An interaction was defined to be any point at which contact was evident between two beads.

Pass 3: The total number of beads in aggregates and the number of beads in aggregates lacking heterotopic interactions were counted.

\section{Acknowledgements}

We thank EPSRC for support of this work through the ROPA and Catalysis Initiatives, and Prof. Tim Claridge for advice on NMR work. Prof. Howard Colquhoun and Prof. Jim Thomas made very valuable comments on the draft manuscript.

\section{References}

1. Bunton, C. A. Prog. Solid State Chem. 1973, 8, 239-81.

https://doi.org/10.1016/0079-6786(73)90008-3

2. Cordes, E. H. Pure Appl. Chem. 1978, 50, 617-25.

https://doi.org/10.1351/pac197850070617

3. Brown, J. M.; Bunton, C. A. J. Chem. Soc., Chem. Commun. 1974, 969-71.

https://doi.org/10.1039/c39740000969

4. Brown, J. M.; Bunton, C. A.; Diaz, S. J. Chem. Soc., Chem. Commun, 1974, 971-2.

https://doi.org/10.1039/c39740000971

5. Brown, J. M.; Elliott, R. L.; Griggs, C. G.; Helmchen, G.; Nill, G. Angew. Chem. Int. Ed. 1981, 20, 890-892. https://doi.org/10.1002/anie.198108901

6. Brown, J. M.; Schofield, J. D. J. Chem. Soc., Chem. Commun. 1975, 434-5.

https://doi.org/10.1039/c39750000434

7. Brown, J. M.; Jenkins, J. A. J. Chem. Soc., Chem. Commun. 1976, 458-9.

https://doi.org/10.1039/c39760000458

8. d'Incan, I.; Viout,P. Tetrahedron, 1975, 31, 159.

https://doi.org/10.1016/0040-4020(75)85011-3

9. Cinouini, M.; Colonna, S.; Molinari, H.; Montanari, F.; Tundo, P. J. Chem. Soc., Chem. Commun. 1976, 394-6. https://doi.org/10.1039/c39760000394

10. Tomoi, M.; Ikeda, M.; Kakiuchi, H. Tetrahedron Lett. 1978, 3757-8. 
https://doi.org/10.1016/S0040-4039(01)95051-8

11. Molinari, H.; Montanari, F.; Quici, S.; Tundo, P. J. Am. Chem. Soc. 1979, 101, 3920-7. https://doi.org/10.1021/ja00508a033

12. Brown, J. M.; Molinari, H. Tetrahedron Lett. 1979, 2933-6. https://doi.org/10.1016/S0040-4039(01)86456-X

13. Hodge, P. Chem. Soc. Rev. 1997, 26, 417-424. https://doi.org/10.1039/cs9972600417

14. Frechet, J. M. J. Tetrahedron, 1981, 37, 663-83. https://doi.org/10.1016/S0040-4020(01)97683-5

15. Brown, J. M.; Ramsden, J. A. Chem. Commun., 1996, 2117-2118. https://doi.org/10.1039/cc9960002117

16. Thomas, I. P.; Ramsden, J. A.; Kovacs, T. Z.; Brown, J. M. Chem. Commun., 1999, 1507-1508. https://doi.org/10.1039/a901643k

17. Chidsey, C. E. D.; Loiacono, D. N. Langmuir 1990, 6, 682-91. https://doi.org/10.1021/la00093a026

18. Lyerla, J. R., Jr.; Levy, G. C. Top. Carbon-13 NMR Spectrosc. 1974, 1, 79-148.

19. Nery, H.; Soederman, O.; Canet, D.; Walderhaug, H.; Lindman, B. J. Phys. Chem. 1986, 90, 5802-8. https://doi.org/10.1021/j100280a066

20. Ribeiro, A. A.; Dennis, E. A. J. Phys. Chem. 1977, 81, 957-63. https://doi.org/10.1021/j100525a006

21. Hirai, Y.; Ito, T.; Imamura, Y. Bull. Chem. Soc. Jpn. 1978, 51, 677-81. https://doi.org/10.1246/bcsj.51.677

22. Carpino, L. A.; Sadat-Aalaee, D.; Chao, H. G.; DeSelms, R. H. J. Am. Chem. Soc. 1990, 112 (26), $9651-2$. https://doi.org/10.1021/ja00182a041

23. Pedroso, E.; Grandas, A.; De las Heras, X.; Eritja, R.; Giralt, E. Tetrahedron Lett. 1986, 27, 743-6. https://doi.org/10.1016/S0040-4039(00)84089-7

24. Breed, P. G.; Ramsden, J. A.; Brown, J. M. Can. J. Chem. 2001, 79, 1049-1057. https://doi.org/10.1139/v01-045

25. Gotfredsen, C. H.; Grotli, M.; Willert, M.; Meldal, M.; Duus, J. O. Perkin 1, 2000, 1167-1171. https://doi.org/10.1039/a906835j

26. Grotli, M.; Gotfredsen, C. H.; Rademann, J.; Buchardt, J.; Clark, A. J.; Duus, J. O.; Meldal, M. J. Comb. Chem. 2000, 2, 108-119. https://doi.org/10.1021/cc990048c

27. Cao, X.; Mjalli, A. M. M. Tetrahedron Lett. 1996, 37, 6073-6076. https://doi.org/10.1016/0040-4039(96)01294-4

28. Lowik, D. W. P. M.; Weingarten, M. D.; Broekema, M.; Brouwer, A. J.; Still, W. C.; Liskamp, R. M. J. Angew. Chem., Int. Ed. 1998, 37, 1846-1850. https://doi.org/10.1002/(SICI)1521-3773(19980803)37:13/14<1846::AID-ANIE1846>3.0.CO;2-E

29. Hermentin, P.; Doenges, R.; Gronski, P.; Bosslet, K.; Kraemer, H. P.; Hoffmann, D.; Zilg, H.; Steinstraesser, A.; Schwarz, A.; et. al. Bioconjugate Chem. 1990, 1, 100-7. https://doi.org/10.1021/bc00002a003

30. Bain, C. D.; Whitesides, G. M. Science, 1988, 240, 62-3. 
https://doi.org/10.1126/science.240.4848.62

31. Kolb, H. C.; Finn, M. G.; Sharpless, K. B. Angew. Chem., Int. Ed. 2001, 40, 2004-2021. https://doi.org/10.1002/1521-3773(20010601)40:11<2004::AID-ANIE2004>3.0.CO;2-5

32. Rostovtsev, V. V.; Green, L. G.; Fokin, V. V.; Sharpless, K. B. Angew. Chem., Int. Ed. 2002, 41, $2596-2599$. https://doi.org/10.1002/1521-3773(20020715)41:14<2596::AID-ANIE2596>3.0.CO;2-4

33. Kolb, H. C.; Sharpless, K. B. Drug Discovery Today 2003, 8, 1128-1137. https://doi.org/10.1016/S1359-6446(03)02933-7

34. Kaval, N.; Ermolat'ev, D.; Appukkuttan, P.; Dehaen, W.; Kappe, C. O.; Van der Eycken, E. J. Comb. Chem. 2005, 7, 490-502. https://doi.org/10.1021/cc0498377

35. Graham, D.; Enright, A. Curr. Org. Synth. 2006, 3, 9-17. https://doi.org/10.2174/157017906775473920

36. Wu, P.; Malkoch, M.; Hunt, J. N.; Vestberg, R.; Kaltgrad, E.; Finn, M. G.; Fokin, V. V.; Sharpless, K. B.; Hawker, C. J. Chem. Commun. 2005, 5775-5777. https://doi.org/10.1039/b512021g

37. Kim, T.-D.; Luo, J.; Tian, Y.; Ka, J.-W.; Tucker, N. M.; Haller, M.; Kang, J.-W.; Jen, A. K. Y. Macromolecules 2006, 39 (5), 1676-1680. https://doi.org/10.1021/ma052087k

38. Inglis, A. J.; Sinnwell, S.; Davis, T. P.; Barner-Kowollik, C.; Stenzel, M. H. Macromolecules, 2008, 41, 41204126.

https://doi.org/10.1021/ma8002328

39. Gacal, B.; Durmaz, H.; Tasdelen, M. A.; Hizal, G.; Tunca, U.; Yagci, Y.; Demirel, A. L. Macromolecules 2006, 39, 5330-5336.

https://doi.org/10.1021/ma060690c

40. Durmaz, H.; Dag, A.; Hizal, G.; Tunca, U. J. Polym. Sci., Part A: Polym. Chem. 2011, 49, 1195-1200. https://doi.org/10.1002/pola.24537 